\title{
Correlation of Oxygenation and Radiographic Assessment of Lung Edema (RALE) Score to Lung Ultrasound Score (LUS) in Acute Respiratory Distress Syndrome (ARDS) Patients in the Intensive Care Unit
}

Pratibha Todur MScRT ${ }^{1}$, Souvik Chaudhuri FNB Critical Care, MD, MBBS², Vedaghosh Amara FNB Critical Care, DNB, $\mathrm{MBBS}^{2}$, Srikant N MDS, BDS 3 , Prabha Prakash BDS ${ }^{4}$

P Todur, S Chaudhuri, V Amara., N Srikant, P Prakash. Correlation of Oxygenation and Radiographic Assessment of Lung Edema (RALE) Score to Lung Ultrasound Score (LUS) in Acute Respiratory Distress Syndrome (ARDS) Patients in the Intensive Care Unit. Can J Respir Ther 2021;57:53-59. doi: 10.29390/cjrt-2020-063.

\begin{abstract}
Background: Lung ultrasound score (LUS) as well as radiographic assessment of lung edema (RALE) score as calculated from chest radiography (CXR) have been applied to assess Acute Respiratory Distress Syndrome (ARDS) severity. CXRs, which are frequently performed in ARDS patients, pose a greater risk of radiation exposure to patients and health care staff.

Aims and objectives: The aim of the study was to evaluate if LUS had a better correlation to oxygenation $\left(\mathrm{PaO}_{2} / \mathrm{FiO}_{2}\right)$ compared with the RALE score in ARDS patients. We also aimed to analyse if there was a correlation between RALE score and LUS. We wanted to determine the LUS and RALE score cut-off, which could predict a prolonged length of intensive care unit (ICU) stay ( $\geq 10$ days) and survival.

Methods: Thirty-seven patients aged above 18 years with ARDS as per Berlin definition and admitted to the ICU were included in the study. It was a retro-

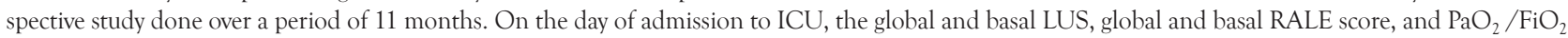
were recorded. Outcome and days of ICU stay were noted.

Results: Global LUS score and $\mathrm{PaO}_{2} / \mathrm{FiO}_{2}$ showed the best negative correlation $(r=-0.491)$, which was significant $(p=0.002)$, followed by global RALE score and $\mathrm{PaO}_{2} / \mathrm{FiO}_{2}(r=-0.422, p=0.009)$. Basal LUS and $\mathrm{PaO}_{2} / \mathrm{FiO}_{2}$ also had moderate negative correlation $(r=-0.334, p=0.043)$ followed by basal RALE score and $\mathrm{PaO}_{2} / \mathrm{FiO}_{2}(r=-0.34, p=0.039)$. Global RALE score and global LUS did not show a significant correlation. Similarly, there was no significant correlation between basal RALE score and basal LUS. Global and basal LUS as well as global and basal RALE score were not beneficial in predicting either a prolonged length of ICU stay or survival as the area under curve was low.

Conclusion: In ARDS patients, global LUS had the best correlation to oxygenation $\left(\mathrm{PaO}_{2} / \mathrm{FiO}_{2}\right)$, followed by global RALE score. Basal LUS and basal RALE score also had moderate correlation to oxygenation. However, there was no significant correlation between global LUS and global RALE score as well as between basal LUS and basal RALE score. Global and basal LUS as well as global and basal RALE scores were not able to predict a prolonged ICU stay or survival in ARDS patients.
\end{abstract}

Key Words: Lung Ultrasound Score (LUS); Radiographic Assessment of Lung Edema (RALE) score; oxygenation; prolonged ICU stay; predictor of survival

\section{INTRODUCTION}

Bedside lung ultrasound examination (LUE) has become an indispensable tool for the clinician. It is extremely beneficial to not only diagnose, but also treat and prognosticate patients of Acute Respiratory Distress Syndrome (ARDS) [1]. Such was the significance of LUE that a modified Berlin definition of ARDS has recommended that LUE be used as a tool to recognize bilateral lung opacities and enable evaluation of ARDS and its outcome in resource-constrained settings [2]. Even though the utility of LUE is well known, very few studies have evaluated whether lung ultrasound score (LUS) has good correlation to oxygenation and chest radiographic score in ARDS patients and if one can predict length of intensive care unit (ICU) stay or survival from cut-off scores. The Radiographic
Assessment of Lung Edema (RALE) was described to gauge the extent as well as the density of alveolar opacities on the chest radiograph (CXR) [3]. RALE score has been shown to independently predict ARDS severity in terms of oxygenation as well as outcomes [3]. However, CXR has its own disadvantages. X-rays are an important source of man-made radiation exposure, and is an important proven carcinogen [4]. Cancers due to radiation are not evident until about two decades post exposure, thus having an insidious yet detrimental effect [4]. Women, especially if pregnant, and children are more vulnerable to the harmful effects of radiations due to CXR [5, 6]. Although the dose of harmful radiation from a single CXR is minimal, the cumulative exposure of this frequently done investigation poses a significant risk, both to patients and ICU health workers [7, 8]. It

\footnotetext{
${ }^{1}$ Department of Respiratory Therapy, Manipal College of Health Professions, Manipal Academy of Higher Education, Manipal, Karnataka, India

${ }^{2}$ Department of Critical Care Medicine, Kasturba Medical College, Manipal Academy of Higher Education, Manipal, Karnataka, India

${ }^{3}$ Department of Oral Pathology and Microbiology, Manipal College of Dental Sciences, Mangalore, Karnataka, India

${ }^{4}$ Prasanna School of Public Health, Manipal Academy of Higher Education, Manipal, Karnataka, India

Correspondence: Souvik Chaudhuri, Department of Critical Care Medicine, Kasturba Medical College, Manipal Academy of Higher Education, Manipal, Karnataka, India. Tel: +91 9937178620, E-mail: souvik.chaudhuri@manipal.edu
}

Published online at https://www.cjrt.ca on 19 May 2021 
may also lead to difficulty in controlling highly infectious respiratory diseases due to entry of X-ray technicians repeatedly in the ICUs and the films being subsequently taken outside. With these concerns, we wanted to evaluate whether total or global LUS(G) and basal LUS(B) have a good correlation to oxygenation $\left(\mathrm{PaO}_{2} / \mathrm{FiO}_{2}\right)$. For the LUE, each hemithorax was divided six regions, as anterior, lateral, and posterior regions, which were in-turn subdivided divided into superior and inferior regions [9]. The LUS $(G)$ was calculated by the summation of scores in all the 12 regions bilaterally (Figure 1) [9]. The LUS(B) was calculated by the addition of scores in the inferior regions of the anterior, lateral, and posterior regions bilaterally (total of six). If a good correlation of LUS to $\mathrm{PaO}_{2} / \mathrm{FiO}_{2}$ is found, we can restrict the CXR performed on ARDS patients and rather routinely use the bedside LUE. We also aimed to evaluate whether there was a correlation between the LUS and the RALE score in ARDS patients, and also determine if we could determine cut-off values for the LUS $(G)$, LUS(B), RALE(G) score and RALE(B) score to predict prolonged ICU stay and survival. A prolonged ICU stay was defined as being equal to or longer than 10 days [10].

\section{MATERIALS AND METHODS}

Thirty-seven patients aged above 18 years and diagnosed with ARDS as per Berlin definition and admitted to the ICU were included in the study. This was a single-centre retrospective study carried out over a period 11 months from November 2019 to September 2020. Institution Research Committee (IRC) and Institution Ethical Committee (IEC) approval (IEC 565/2020) was taken prior to commencement of study.

LUS are not usually recorded in ICU charts, even though the findings of ultrasound examination are described. However, we wanted accurate, elaborate, and reliable data of the LUS in ARDS patients, which was only feasible after using the LUS recorded from another trial as database and then comparing to the RALE scores calculated after retrieving their CXR. The LUS of the 12 zones of examination was taken from the database of 37 ARDS patients from ongoing study registered in Clinical Trials Registry-India (CTRI) (CTRI/2019/11/021857), which had similar inclusion and exclusion criteria but different aims and objectives. Written consent was obtained from the primary investigator of the above mentioned trial, who is a part of this study also. A total of 37 patients of ARDS as per Berlin Definition were recruited for the study [11]. Patients above 18 years admitted to critical care unit and who were on noninvasive ventilation (NIV) or invasive mechanical ventilation (IMV) were included for the study. Patients with thoracic injuries, patients with thoracic surgeries in the past, and those with lung malignancies were excluded. The LUS and RALE score were calculated on the day of admission to ICU. LUE was done using a curvilinear probe and Philips Ultrasound Solutions machine. The recruitment and methodology of the participants in study is explained in Figure 1.

Each hemithorax was divided into anterior (between anterior axillary line to sternal margin), lateral (between anterior and posterior axillary lines), and posterior regions (between posterior axillary line to as far the ultrasound probe may be placed by slightly tilting a supine patient). From cranial to caudal direction, each hemithorax was divided into two regions, superior and inferior. A total of 12 regions in both lungs is examined $[9,12]$. The total LUS of the 12 regions bilaterally was referred to as LUS(G). The LUS of basal lung regions (bilaterally inferior regions anteriorly, laterally and posteriorly) was recorded separately as LUS(B). The lung regions that were examined were as depicted in Figure 2 [9].

The amount of lung aeration loss was calculated using the validated LUS $[9,12]$. The LUS ranged from 0 to 36 points, adding all points in 12 lung zones bilaterally $[9,12]$. The scoring was done as shown in Table 1 [9].

The CXR of the same day as the LUS analysis was evaluated for calculating the RALE score [3]. The CXR is divided into four quadrants, upper and lower on right and left side. The minimum RALE score is 0 (no infiltrates) and the maximum RALE score is 48 (dense consolidation in $>75 \%$ of each quadrant). The calculation is shown in Table 2 [3]. The RALE score of the right and left lower quadrants were recorded separately as RALE(B) score and RALE(G) score.

\section{Data collection}

Details of age, gender, Acute Physiology and Chronic Health Evaluation (APACHE II) score, Sequential Organ Failure Assessment (SOFA) score,

\section{FIGURE 1}

The 12 regions of lung ultrasound examination.

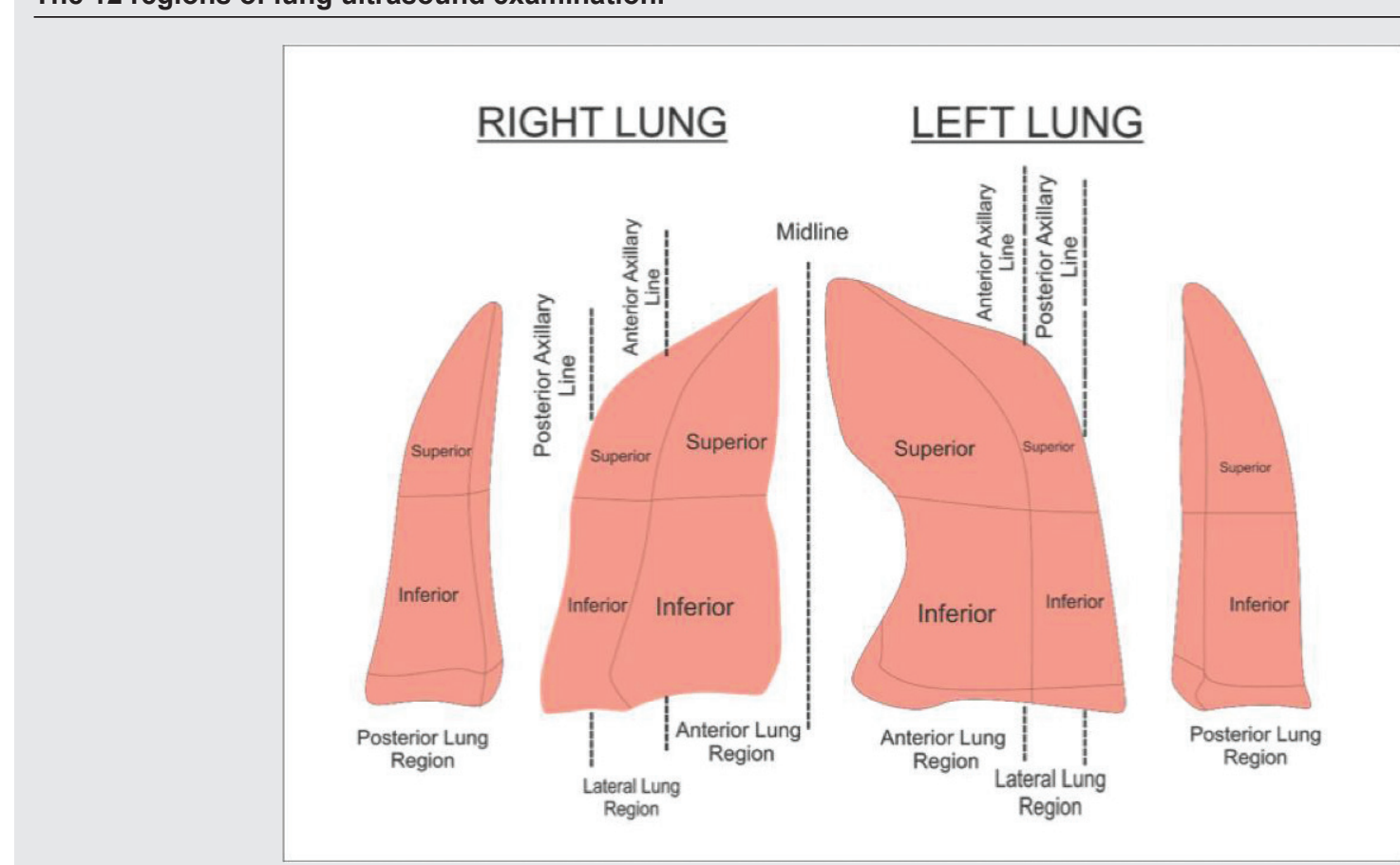




\section{FIGURE 2}

The recruitment and methodology of the participants in study.

As per ICU records: Patients diagnosed with ARDS as per Berlin criteria were noted

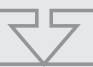

Patient medical records: Screened for inclusion and exclusion criteria and enrolled to study

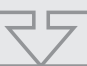

Data noted from records: Age, gender, $\mathrm{PaO}_{2} / \mathrm{FiO}_{2}$, APACHE II score, SOFA score, serum creatinine, mode of respiratory support (Non-invasive ventilation/ Invasive mechanical ventilation), length of ICU stay, outcome of ICU stay,

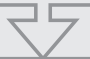

Lung ultrasound score (global): data extracted from a previously approved study by obtaining lung ultrasound examinationof 12 lung regions bilaterally (6 regions on each side)

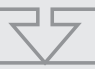

Lung ultrasound score (basal): Lung ultrasoundscore of the 6 basal regions bilaterally (3 basal regions on each side) was recorded separately

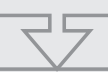

The chest radiograph: Performed on the same day either just prior to or just after the lung ultrasound evaluation was analysed for RALE scoring (Data extracted from Picture Archives and Communication Systems (InstaRISPACS) of the hospital)

TABLE 1

Calculation of Lung Ultrasound Score (LUS) as per pattern of findings during lung ultrasound examination

\begin{tabular}{lll}
\hline Point for each lung zone & Degree of lung aeration & Pattern \\
\hline 0 point & Normal & A lines \\
1 point & Moderate loss & Well-separated B \\
& & lines \\
2 point & Severe loss & Coalescent B lines \\
3 point & Complete loss & Lung consolidation \\
\hline
\end{tabular}

oxygenation in terms of partial pressure of oxygen to fraction of inspired oxygen $\left(\mathrm{PaO}_{2} / \mathrm{FiO}_{2}\right)$, creatinine values and evidence of acute kidney injury (AKI), co-morbidities and days from hospital to ICU admission were recorded. LUS(G), LUS(B), RALE(G) score, and RALE(B) score, days of ICU stay, and outcome of ICU stay were recorded.

Statistical analysis

Data were analysed using the SPSS software version 19 and the EZR software version 1.53 , to calculate mean and standard deviation (SD) of the continuous variables.

LUS(G), LUS(B), RALE(G) score, and RALE(B) score were correlated to $\mathrm{PaO}_{2} / \mathrm{FiO}_{2}$; the LUS(G) and RALE(G) score as well as the LUS(B) and RALE(B) score were also correlated to one another.

Pearson's correlation coefficient test was done for correlation of variables and its interpretation. Positive correlation means as one parameter value increases the other also increases. Negative correlation means as one parameter increases the other decreases. Pearson's correlation, $r<0.3$ was considered weak correlation, $r$ between 0.3-0.69 was considered moderate correlation and $r>0.7$ was considered strong correlation. A p value $<0.05$ was considered statistically significant. The ability of LUS(G), LUS (B), RALE (G) and RALE (B) score cut-offs to predict days of ICU stay and survival was done using the receiver operating characteristic (ROC) curve analysis after calculating area under the curve
TABLE 2

Method of calculation of Radiographic Assessment of Lung Edema (RALE) score from chest radiography

\begin{tabular}{lc}
\hline Consolidation & \\
\hline $\begin{array}{l}\text { Consolidation score } \\
\text { (Con) }\end{array}$ & Extent of alveolar opacities \\
\hline 0 & None \\
1 & $<25 \%$ \\
2 & $25 \%-50 \%$ \\
3 & $50 \%-75 \%$ \\
4 & $>75 \%$ \\
\hline
\end{tabular}

\section{Density}

\begin{tabular}{lc}
\hline Density score (Den) & Density of alveolar opacities \\
\hline 1 & Hazy \\
2 & Moderate \\
3 & Dense
\end{tabular}

\section{Final RALE score}

Right lung

Upper quadrant

Con $\times$ Den $=$ Q1 score

Lower quadrant

Con $\times$ Den $=$ Q2 score

Left lung
Upper quadrant
Con $\times$ Den $=\mathrm{Q} 3$ score
Lower quadrant
Con $\times$ Den $=\mathrm{Q} 4$ score

Total RALE score $=\mathrm{Q} 1$ score $+\mathrm{Q} 2$ score $+\mathrm{Q} 3$ score $+\mathrm{Q} 4$ score

(AUC). Log Rank (Mantel-Cox) test was done to analyze equality of survival distributions for various cut-off scores for $L U S(G)$, LUS(B), RALE(G), and RALE(B) for predicting prolonged ICU stay and survival.

\section{RESULTS}

The demographic characteristics and the other variables were as depicted in Table 3. 
Regarding the highest correlation to oxygenation, LUS $(G)$ and $\mathrm{PaO}_{2} / \mathrm{FiO}_{2}$ showed the best correlation. There was moderate negative correlation, which was significant. $(r=-0.491, p=0.002)$. RALE(G) score and $\mathrm{PaO}_{2} / \mathrm{FiO}_{2}$ also revealed a moderate negative correlation, which was significant $(r=-0.422, p=0.009)$. LUS(B) and $\mathrm{PaO}_{2} / \mathrm{FiO}_{2}$ also had significant negative correlation $(r=-0.334, p=0.043)$ followed by RALE(B) score and $\mathrm{PaO}_{2} / \mathrm{FiO}_{2}(r=-0.34, p=0.039)$ (Table 4). However, there was no significant correlation between LUS $(G)$ and RALE $(G)$ score and between LUS(B) and RALE(B) score as shown in Table 4. The AUC of LUS $(G)$ and LUS(B) in predicting a prolonged ICU stay was low (cut-off 24.5, AUC 0.607 and cut-off 13.5, AUC 0.528, respectively) which is depicted in Figure 3. The AUC of RALE(G) score and RALE(B) score in predicting a prolonged ICU stay was also low (cutoff 21, AUC 0.475 and cut-off 11.5, AUC 0.521, respectively). Similarly, the AUC of LUS(G) and LUS(B) in predicting survival was also low (cutoff 24.5, AUC 0.540 and cut-off 13.5 with AUC 0.402 , respectively) as shown in Figure 4. The AUC of RALE $(G)$ score and RALE(B) score to predict survival was also low, being 0.498 and 0.567 , respectively, at cutoff 21 for RALE(G) score and 11.5 for RALE(B) score as depicted in Figure 4. There was no correlation between the LUS, RALE score, and incidence of AKI in ARDS patients as seen in Table 4. The linear correlation patterns of the various parameters analysed in the study is depicted in the scatter plots in Figure 5.

\section{DISCUSSION}

LUS has become a vital tool in diagnosing various respiratory disorders and it indicates the "degree of aeration" of the lungs [13]. The appearance

\section{TABLE 3}

\section{Depiction of demographic and other study variables}

\begin{tabular}{lc}
\hline Variable & $\begin{array}{c}\text { Baseline character }(\boldsymbol{n}=\mathbf{3 7}), \text { mean } \mathbf{\pm} \\
\text { standard deviation (SD) }\end{array}$ \\
\hline Age (years) & $51.43 \pm 14.7$ \\
Gender & $62.2 \%$ \\
Male (\%) & $37.8 \%$ \\
Female (\%) & $15.38 \pm 7.36$ \\
APACHE score & $9.30 \pm 4.31$ \\
SOFA score & $170.14 \pm 60.48$ \\
PaO $/$ FiO & ratio \\
LUS(G) & $24.51 \pm 4.27$ \\
LUS(B) & $13.24 \pm 1.98$ \\
RALE(G) & $20.73 \pm 9.77$ \\
RALE(B) & $13.54 \pm 6.9$ \\
\hline
\end{tabular}

Note: APACHE, Acute Physiology and Chronic Health Evaluation; SOFA, Sequential Organ Failure Assessment; LUS(G), total/global lung ultrasound score; LUS(B); basal lung ultrasound score; $R A L E(G)$, total/global radiographic assessment of lung edema; $R A L E(B)$, basal radiographic assessment of lung edema; $\mathrm{PaO}_{2} / \mathrm{FiO}_{2}$, ratio of partial pressure of oxygen in arterial blood to fraction of inspired oxygen. of "B-lines" in LUE has been shown to indicate reduction in lung aeration [14]. It has been shown in literature that this reduction in aeration as evidenced by the number of lung regions with B-lines correlated with a decrease in $\mathrm{PaO}_{2} / \mathrm{FiO}_{2}$ in post-surgical patients [14]. However, to the best of our knowledge, the correlation of an objectively evaluated LUS and RALE score to $\mathrm{PaO}_{2} / \mathrm{FiO}_{2}$ in ARDS patients has not been done. Lung ultrasound has been shown in a previous study to have a comparative performance with chest radiography and estimate the extent of lung injury [15]. But the study used the characteristic descriptive findings of the lung ultrasound and CXR, rather than an objectively defined scoring system. Thus, we wanted to evaluate which score out of LUS(G), LUS(B), RALE(G) score, and RALE(B) score correlated best with oxygenation $\left(\mathrm{PaO}_{2} / \mathrm{FiO}_{2}\right)$. We also tried to analyse if there was good correlation between LUS and RALE score and assess the cut-offs for LUS(G), LUS(B), RALE(G) score, and RALE(B) score to predict prolonged length of stay in ICU. LUS has been shown to independently relate to 28-day mortality in shock patients in the ICU [16].

We did a separate scoring for the basal lung regions (anterior, lateral, and posterior). It has been shown that LUS of the posterior regions is the major contributor to the total score [17]. However, analysis of the basal lung regions to oxygenation and any particular CXR score is not reported.

The lungs are not homogenous in shape but are conical, with more alveolar units at the bases [18]. Thus, we chose to do a separate basal lung ultrasound and basal RALE scoring and assess correlation to $\mathrm{PaO}_{2} / \mathrm{FiO}_{2}$.

In our study, the LUS(G) had the best negative correlation to $\mathrm{PaO}_{2} /$ $\mathrm{FiO}_{2}$, followed by RALE(G) score, LUS(B), and then RALE(B) score. LUS was also found to have good correlation to $\mathrm{PaO}_{2} / \mathrm{FiO}_{2}$ in a previous study in shock patients [16]. This is significant because the clinician can just perform the LUS to analyse improving or worsening oxygenation, rather than repeated withdrawing arterial blood for analysis (ABG) of oxygenation. LUS is more informative than $\mathrm{PaO}_{2} / \mathrm{FiO}_{2}$ about worsening clinical condition [16]. LUS enables the clinician to know real-time lung aeration and to plan treatment according to those specific pathological changes [16]. Since the LUS $(G)$ had a better correlation to oxygenation than the RALE (G) score, as clinicians we can restrict the CXR performed in ARDS patients, and rather repeat bedside LUE for a better analysis of the lung aeration and oxygenation. This will prevent the adverse effects of radiations on the patients.

LUS(B) separately also has a moderate negative correlation to oxygenation. This can be explained physiologically as well, because the maximum number of alveolar units is actually in the basal lung regions due to the unique conical shape of the lungs. We wanted to analyze a cut-off score for predicting prolonged ICU stay as well as survival in ARDS patients. However, with the cut-off scores of 24.5 for LUS(G), 13.5 for LUS(B), 21 for RALE(G) score, and 11.5 RALE(B) score, the respective AUC for predicting prolonged ICU stay and survival was low. Thus, we were unable to predict the length of ICU stay or the chance of survival using any score for lung ultrasound and RALE. A previous study had shown LUS < 24.3 for lesser ICU stay and favourable outcome [19]. The authors in the study had reported LUS $24.3 \pm 3.8$ in the death

\section{TABLE 4}

Correlation of the various parameters analysed in the study $(r<0.3$ was considered weak correlation, $r$ between $0.3-0.69$ was considered moderate correlation and $r>0.7$ was considered strong correlation, $p$-value $<0.05$ is significant)

\begin{tabular}{|c|c|c|c|c|}
\hline Serial number & Parameters being correlated & $N=37$ & Correlation $(r)$ & $p$-value \\
\hline 1 & LUS(G) and RALE(G) score & 37 & 0.259 & 0.122 \\
\hline 2 & LUS(B) and RALE(B) score & 37 & -0.038 & 0.823 \\
\hline 3 & $\mathrm{PaO}_{2} / \mathrm{FiO}_{2}$ and $\mathrm{LUS}(\mathrm{G})$ & 37 & -0.491 & 0.002 \\
\hline 4 & $\mathrm{PaO}_{2} / \mathrm{FiO}_{2}$ and LUS(B) & 37 & -0.334 & 0.043 \\
\hline 5 & $\mathrm{PaO}_{2} / \mathrm{FiO}_{2}$ and $\mathrm{RALE}(\mathrm{G})$ & 37 & -0.422 & 0.009 \\
\hline 6 & $\mathrm{PaO}_{2} / \mathrm{FiO}_{2}$ and $\mathrm{RALE}(\mathrm{B})$ & 37 & -0.34 & 0.039 \\
\hline 7 & LUS(G) and AKI & 37 & 0.223 & 0.184 \\
\hline 8 & LUS(B) and AKI & 37 & 0.137 & 0.419 \\
\hline 9 & $\operatorname{RALE}(\mathrm{G})$ score and AKI & 37 & 0.14 & 0.401 \\
\hline 10 & RALE(B) and AKI & 37 & 0.125 & 0.46 \\
\hline
\end{tabular}

Note: LUS(G), total/global lung ultrasound score; LUS(B), basal lung ultrasound score; RALE(G), total/global radiographic assessment of lung edema; RALE(B), basal radiographic assessment of lung edema; $\mathrm{PaO}_{2} / \mathrm{FiO}_{2}$, ratio of partial pressure of oxygen in arterial blood to fraction of inspired oxygen; AKI, acute kidney injury. 


\section{FIGURE 3}

The receiver operating characteristics (ROC) curve depicting the area under curve (AUC) for LUS(G), LUS(B), RALE(G), RALE(B) in predicting a prolonged ICU stay. LUS = lung ultrasound score, RALE = radiographic assessment of lung edema, $\mathbf{G}=$ global, $\mathbf{B}=$ basal.

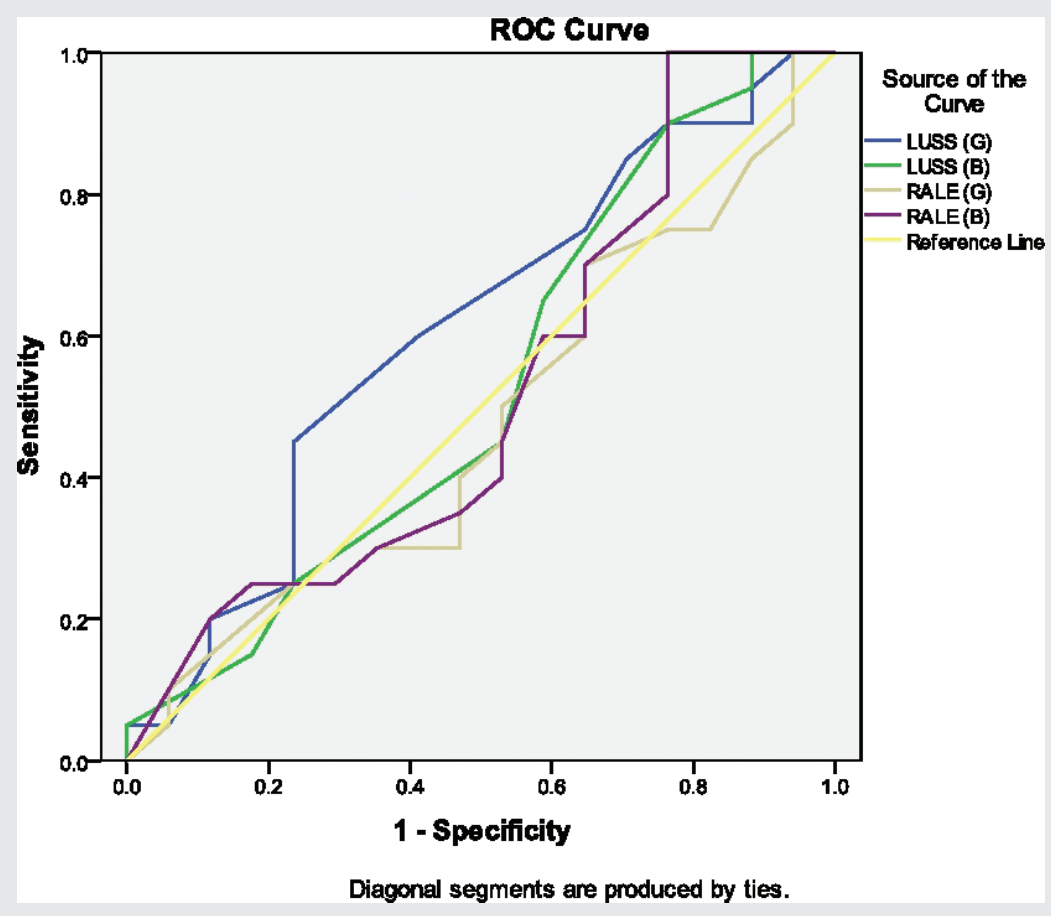

\section{FIGURE 4}

The receiver operating characteristics (ROC) curve depicting the area under curve (AUC) for LUS(G), LUS (B), RALE(G), RALE(B) in predicting survival. LUS = lung ultrasound score, $R A L E=$ radiographic assessment of lung edema, $G=g l o b a l$, $B=$ basal.

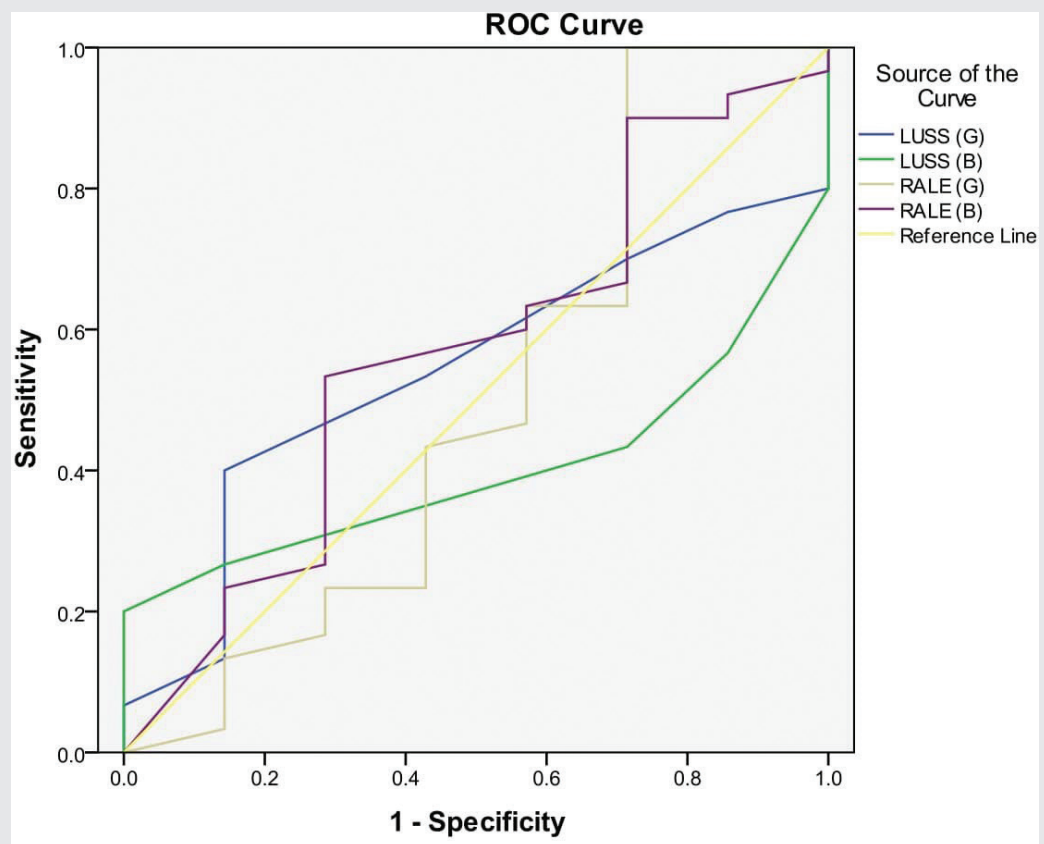

Diagonal segments are produced by ties. 


\section{FIGURE 5.}

\section{Scatter diagram depicting the correlation between different parameters in the study.}

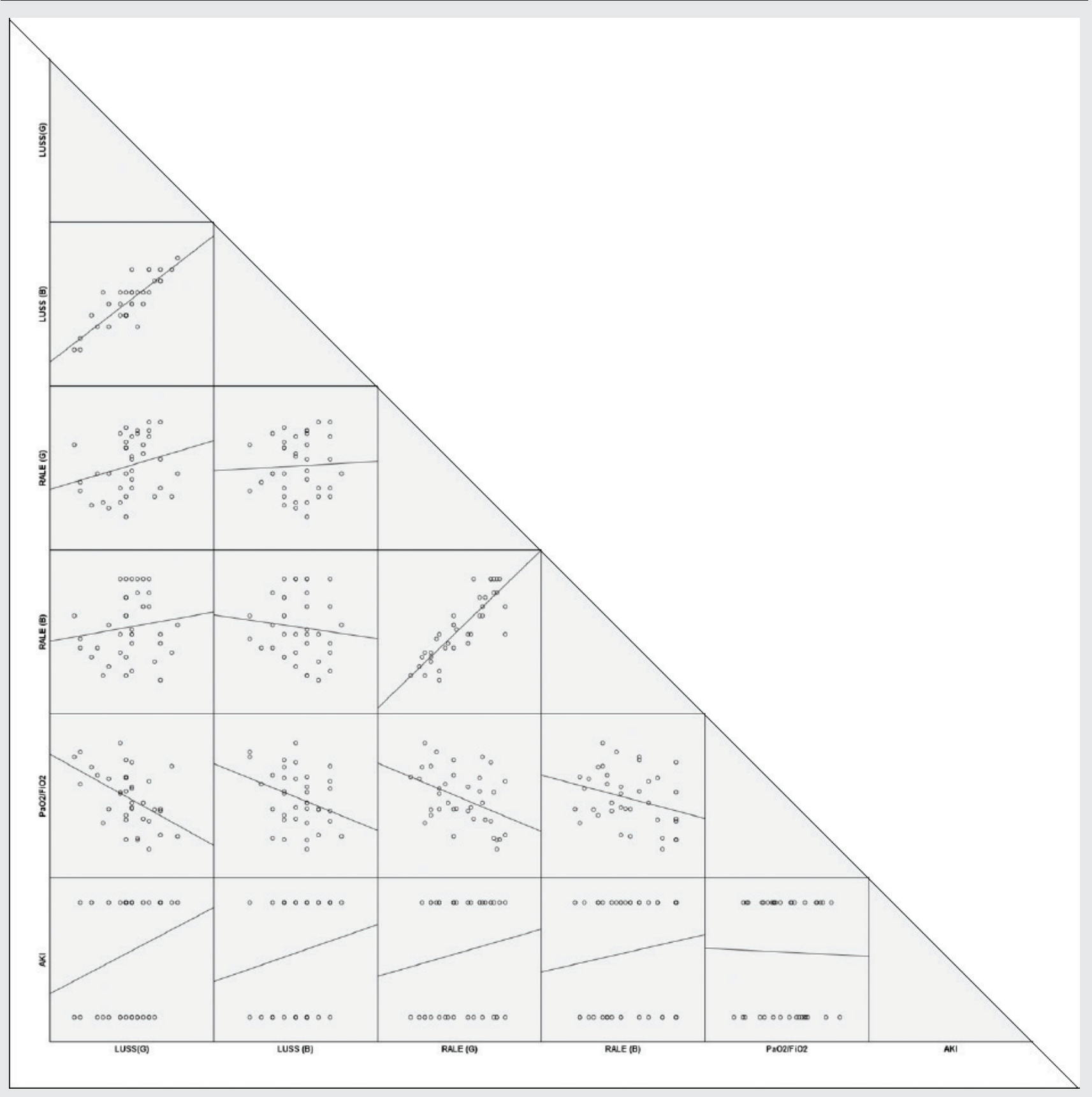

group and $12.7 \pm 2.9$ in the survival group [19]. In our study we were unable to predict cut-off score of LUS $(G)$ to predict survival.

The LUE in ARDS patients has been shown to identify patients who are having a higher risk of death, even though patients did not fulfil the CXR criteria prescribed as per Berlin definition [20]. However, in the study, a scoring system was not used in ARDS patients, rather findings of the LUE in terms of consolidation and B-lines were described [20]. In the study done on ARDS patients with COVID-19 pneumonia, the average LUS on admission was 22 [21]. It was similar to that of our study, wherein mean and SD of the LUS $(G)$ was $24.51 \pm 4.27$.

In our study, the mean and SD of RALE $(G)$ score was $20.73 \pm 9.77$. In a previous study, the average RALE score for ARDS patients was found to be 23 and that without ARDS was 9 [22].
Significant correlation between RALE score and LUS in mechanically ventilated patients was shown in the study done by Pisani et al. [23]. The authors had concluded that the LUS was in agreement with the RALE score in CXR [23]. However, in the study, there were only 33 out of 144 patients with ARDS, and the linear correlation was depicted more in the non-ARDS patients [23].

This was in contrast to the findings of our study, where there was no significant correlation between the LUS and RALE score in ARDS patients. This can be explained by the fact that changes in lung ultrasound may be identified much earlier than the onset of severe clinical symptoms [24]. Ultrasound has also been shown to be superior to CXR when assessing interstitial pathologies and consolidations [24]. Hence the lack of correlation between the LUS and RALE score could 
have been due to the early, elaborate lung ultrasound findings, which often precede onset of symptoms and reflect the precise changes in a computerized tomography (CT) scan, rather than CXR [24]. The lack of correlation in our study between the LUS and RALE score could also be that lung ultrasound has a much higher sensitivity and specificity in detecting consolidation and diagnosis of pneumonia [25].

LUS(G) and LUS(B) had a more significant correlation to oxygenation than RALE $(G)$ and RALE(B) scores, respectively. LUE is non-ionising, available at the bedside, and may be repeated multiple times by the respiratory therapists and critical care physicians. It is less time consuming compared with the image processing after obtaining a CXR [24]. In our study, we had compared the $\mathrm{PaO}_{2} / \mathrm{FiO}_{2}$ to LUS(G) and LUS(B) as well as RALE $(G)$ and RALE(B). Since LUS $(G)$ showed the best correlation to $\mathrm{PaO}_{2} / \mathrm{FiO}_{2}$, which was better than the correlation of CXR-derived RALE(G) score to $\mathrm{PaO}_{2} / \mathrm{FiO}_{2}$, the utility of lung ultrasound as a better predictor of lung aeration than CXR in ARDS patients has to be emphasized.

We want to propose the utility of LUS(G) and LUS(B) rather than routine daily CXR for radiological assessment of ARDS patients. Frequent $\mathrm{ABG}$ analysis for lung aeration assessment may also be avoided, especially in coagulopathy patients, where we want to prevent repeated arterial pricks. Rather, the clinician can get more information about lung aeration by doing LUS(G) as well as LUS(B).

Limitations of our study include the single-centre retrospective study with a small sample size. The other limitation is that we analysed the LUS and RALE score for each patient only on the day of admission, and the investigator analysing the LUS was not blinded to $\mathrm{PaO}_{2} / \mathrm{FiO}_{2}$

\section{CONCLUSION}

In ARDS patients, the LUS $(G)$ had the best correlation to oxygenation, followed by the RALE(G) score. The LUS(B) and RALE(B) score also had moderate negative correlation to oxygenation. However, there was no significant correlation between $\operatorname{LUS}(G)$ and $\operatorname{RALE}(G)$ score as well as between LUS(B) and RALE(B) score. Particular cut-off scores of LUS(G) and LUS(B) as well as RALE(G) and RALE(B) scores were not able to predict a prolonged length of ICU stay or survival. Further prospective study with a larger sample size has to be conducted to strengthen the findings of present study.

\section{DISCLOSURES}

Funding: This study did not receive any specific grant from funding agencies in the public, commercial, or not-for-profit sectors.

Competing interests: All authors have completed the ICMJE uniform disclosure form at www.icmje.org/coi_disclosure.pdf and declare: no financial relationships with any organizations that might have an interest in the submitted work in the previous 3 years; no other relationships or activities that could appear to have influenced the submitted work.

Ethical approval: It was a retrospective study for which Informed consent was waived off by the Institutional Ethics Committee. Ethical Requirement of Research Ethics Board approval for this project was obtained from Institutional Ethics Committee (IEC 565/2020) after review of the study by Institutional Research Committee.

\section{REFERENCES}

1. Zhou Y, Fan Q, Cavus O, Zhang X. Lung ultrasound: predictor of acute respiratory distress syndrome in intensive care unit patients. Saudi J Anaesth 2018;12(3):457-61. doi: 10.4103/sja.SJA_73_18.

2. Riviello ED, Kiviri W, Twagirumugabe T, et al. Hospital incidence and outcomes of the acute respiratory distress syndrome using the Kigali modification of the Berlin definition. Am J Respir Crit Care Med 2016;193(1):52-9. doi: 10.1164/rccm.201503-0584OC.

3. Warren MA, Zhao Z, Koyama T, et al. Severity scoring of lung oedema on the chest radiograph is associated with clinical outcomes in ARDS. Thorax 2018;73(9):840-6. doi: 10.1136/thoraxjnl-2017-211280.

4. Gargani L, Picano E. The risk of cumulative radiation exposure in chest imaging and the advantage of bedside ultrasound. Crit Ultrasound J 2015;7(4):20-3. doi: 10.1186/s13089-015-0020-x.

5. Picano E, Vañó E, Rehani MM, et al. The appropriate and justified use of medical radiation in cardiovascular imaging: a position document of the ESC Associations of Cardiovascular Imaging, Percutaneous
Cardiovascular Interventions and Electrophysiology. Eur Heart J 2014;35(10):665-72. doi: 10.1093/eurheartj/eht394.

6. Dorfman AL, Fazel R, Sc M, et al. Use of medical imaging procedures with ionizing radiation in children: a population-based study. Arch Pediatr Adolesc Med 2013;165(5):458-64. doi: 10.1001/ archpediatrics.2010.270.

7. Veldkamp WJ, Kroft LJ, Geleijns J. Dose and perceived image quality in chest radiography. Eur J Radiol 2009;72(2):209-17. doi: 10.1016/j. ejrad.2009.05.039.

8. Ganapathy A, Adhikari NKJ, Spiegelman J, Scales DC. Routine chest $\mathrm{x}$-rays in intensive care units: a systematic review and meta-analysis. Crit Care 2012;16(2):R68. Available from: http://ccforum.com/content/16/2/R68 [Accessed 01 December 2020].

9. Bouhemad B, Mongodi S, Via G, Rouquette I. Ultrasound for "Lung Monitoring" of ventilated patients. Anesthesiology 2015 Feb 1;122(2):437-47. doi: 10.1097/ALN.0000000000000558.

10. Chan CL, Ting HW, Huang HT. The definition of a prolonged intensive care unit stay for spontaneous intracerebral hemorrhage patients: an application with national health insurance research database. Biomed Res Int 2014;2014:26-9. doi: 10.1155/2014/891725.

11. ARDS Definition Task Force; Ranieri MV, Rubenfeld GD, Thompson T, et al. Acute respiratory distress syndrome: the Berlin definition. JAMA 2012;307(23):2526-33. doi: 10.1001/jama.2012.5669.

12. Banerjee A, Mehrotra G. Comparison of lung ultrasound-based weaning indices with rapid shallow breathing index: are they helpful? Indian J Crit Care Med 2018;22(6):435-40. doi: 10.4103/ijccm. IJCCM_331_17.

13. Lichtenstein D. Lung ultrasound in the critically ill. Curr Opin Crit Care 2014;20(3):315-22. doi: 10.1097/MCC.0000000000000096.

14. Koh J, Hong J, Kweon T, Park J, Ko E, Kim J. Relationship between $\mathrm{PaO}_{2} /$ $\mathrm{FiO}_{2}$ and number of regions with B-line on transthoracic lung ultrasound: a prospective, observational study. Anesth Pain Med 2019 Apr 30;14:187-92. doi: 10.17085/apm.2019.14.2.187.

15. Lichtenstein D, Goldstein I, Mourgeon E, Cluzel P, Grenier P, Rouby J-J. Comparative diagnostic performances of auscultation, chest radiography, and lung ultrasonography in acute respiratory distress syndrome. Anesthesiology 2004 Jan 1;100(1):9-15. doi: 10.1097/ 00000542-200401000-00006.

16. Yin W, Zou T, Qin Y, et al. Poor lung ultrasound score in shock patients admitted to the ICU is associated with worse outcome. BMC Pulm Med 2019;19(1):1-8. doi: 10.1186/s12890-018-0755-9.

17. Pisani L, Vercesi V, van Tongeren PSI, et al. The diagnostic accuracy for ARDS of global versus regional lung ultrasound scores - a post hoc analysis of an observational study in invasively ventilated ICU patients. Intensive Care Med Exp 2019;7(S1):1-11. doi: 10.1186/s40635-019-0241-6.

18. Gattinoni L, Taccone P, Carlesso E, Marini JJ. Prone position in acute respiratory distress syndrome. Rationale, indications, and limits. Am J Respir Crit Care Med 2013 Dec 1;188(11):1286-93. doi: 10.1164/ rccm.201308-1532CI.

19. Li L, Yang Q, Li L, et al. The value of lung ultrasound score on evaluating clinical severity and prognosis in patients with acute respiratory distress syndrome. Chinese Crit Care Med 2015;27:579-84.

20. See KC, Ong V, Tan YL, Sahagun J, Taculod J. Chest radiography versus lung ultrasound for identification of acute respiratory distress syndrome: a retrospective observational study. Crit Care 2018;22(1):1-9. doi: 10.1186/s13054-018-2105-y.

21. Dargent A, Chatelain E, Kreitmann L, et al. Lung ultrasound score to monitor COVID-19 pneumonia progression in patients with ARDS. PLoS One 2020;15(7 July):1-5. doi: 10.1371/journal.pone.0236312.

22. Zimatore $\mathrm{C}$, Pisani L, Lippolis V, et al. The radiographic assessment of lung edema (RALE) score has excellent diagnostic accuracy for ARDS. Eur Respir J 2019 Sep 28;54(suppl 63):OA3299. Available from: http:// erj.ersjournals.com/content/54/suppl_63/OA3299.abstract [Accessed 01 December 2020]

23. Pisani L, Zimatore C, Vercesi V, et al. Agreement between lung ultrasound and chest X-ray scoring in invasively ventilated ICU patients. Eur Respir J 2019;54(suppl 63). Available from: https://erj.ersjournals.com/ content/54/suppl_63/OA3300 [Accessed 01 December 2020].

24. Liu RB, Tayal VS, Panebianco NL, et al. Ultrasound on the Frontlines of COVID-19: report from an international webinar. Acad Emerg Med 2020;27(6):523-6. doi: 10.1111/acem.14004.

25. Amatya Y, Rupp J, Russell FM, Saunders J, Bales B, House DR. Diagnostic use of lung ultrasound compared to chest radiograph for suspected pneumonia in a resource-limited setting. Int J Emerg Med 2018;11(1). doi: 10.1186/s12245-018-0170-2. 